\title{
Optimising forecasting models for inventory planning
}

\author{
Nikolaos Kourentzes $^{\mathrm{a}, \mathrm{b}, *}$, Juan R. Trapero ${ }^{\mathrm{c}}$, Devon K. Barrow ${ }^{\mathrm{d}}$ \\ ${ }^{a}$ Department of Management Science, Lancaster University Management School, UK. \\ ${ }^{b}$ Skövde Artificial Intelligence Lab, School of Informatics, University of Skövde, Sweden. \\ ${ }^{c}$ Department of Business Administration, Universidad de Castilla-La Mancha, Ciudad \\ Real, Spain \\ ${ }^{d}$ Birmingham Business School, University of Birmingham, Birmingham, UK.
}

\section{Abstract}

Inaccurate forecasts can be costly for company operations, in terms of stockouts and lost sales, or over-stocking, while not meeting service level targets. The forecasting literature, often disjoint from the needs of the forecast users, has focused on providing optimal models in terms of likelihood and various accuracy metrics. However, there is evidence that this does not always lead to better inventory performance, as often the translation between forecast errors and inventory results is not linear. In this study, we consider an approach to parametrising forecasting models by directly considering appropriate inventory metrics and the current inventory policy. We propose a way to combine the competing multiple inventory objectives, i.e. meeting demand, while eliminating excessive stock, and use the resulting cost function to identify

*Correspondance: N Kourentzes, Department of Management Science, Lancaster University Management School, Lancaster, Lancashire, LA1 4YX, UK.

Email addresses: nikolaos@kourentzes.com (Nikolaos Kourentzes), juanramon.trapero@uclm.es (Juan R. Trapero), d.k.barrow@bham.ac.uk (Devon K. Barrow) 
inventory optimal parameters for forecasting models. We evaluate the proposed parametrisation against established alternatives and demonstrate its performance on real data. Furthermore, we explore the connection between forecast accuracy and inventory performance and discuss the extent to which the former is an appropriate proxy of the latter.

Keywords: Forecasting, inventory management, optimisation, likelihood, simulation

\section{Introduction}

In managing inventory it is critical to quantify the demand uncertainty, typically encapsulated in forecasts of the demand over the lead time period. There is an intuitive link between accurate forecasting and meeting the demand. When the demand is unusually high, compared to the expected, i.e. the forecast, it may result in a stockout, while when the demand is below expectations, additional holding costs can arise. Nonetheless, whether a deviation of demand from the expectation leads to a stockout or excess inventory holding costs is conditional on the current stock on hand. Given sufficient stock, a stockout can be averted, or equivalently by holding lower inventory one can avoid unnecessarily high holding costs.

In a realistic setting, where the demand process is unknown, we rely on forecasts to generate approximations of the expected realised demand over the lead time. However, the generation of forecasts is often rather disjoint from the needs of the forecast users. Forecasting methods, such as 
the exponential smoothing method, are typically optimised on the in-sample mean squared errors (MSE), which when used as a cost function for the parameter optimisation leads to optimal forecasts for the mean of the demand process (Gneiting, 2011a). Similarly, when a model is present, we typically use maximum likelihood estimation, which is again based on quadratic errors, and therefore results in optimal forecasts for the mean of the demand process. This ensures unbiased in-sample forecasts, however this does not guarantee good out-of-sample behaviour (Barrow and Kourentzes, 2016), i.e. accurate or unbiased forecasts. Alternative costs have been explored, such as the mean absolute error (MAE, Gardner Jr, 2006), which are more robust against outlying demand events. Absolute errors result in optimal forecasts for the median of the demand process (Gneiting, 2011a). However, forecasts for inventory management need not be solely optimal either on the mean or median of the demand process.

In fact, there are two elements where classical cost functions fail in an inventory management setting. First, they satisfy their conditions in-sample; and second, inventory decisions are conditional on the inventory position and the ordering policy, neither of which are accounted by MSE, MAE or similar. Furthermore, these metrics do not consider asymmetric costs of overand under-stocking and the associated distributional assumptions. The first point connects to the issue of over-fitting, where a sufficiently flexible model may capture parts of the noise in the in-sample data, thereby harming the out-of-sample performance. Assuming a fixed model form, as is the case with 
exponential smoothing, this will be reflected in the model's parameters. If the model form itself is flexible, this may manifest itself both in the terms included in the model and the parameters' values. The degree of difference depends on how close the model is to the underlying data generating process, which for any realistic case is unknown and typically more complex than the forecasting models we use to approximate it. This is a well documented problem and has led to extensive research in shrinkage estimators, such as lasso regression (Tibshirani, 1996). More recently Kourentzes and Trapero (2018) showed that multi-step ahead quadratic cost functions are a form of univariate shrinkage, which will also mitigate the sampling effects on parameter estimation. Nonetheless, these approaches do not address the second limitation discussed above.

In this paper we propose optimising the forecasts directly on the decision variable. Therefore, instead of relying on some metric of divergence between the fitted values of the forecast functions and the observed historical demand, we conduct an inventory simulation and optimise the forecast function parameters, so as to maximise the relevant inventory performance metrics. This follows the ideas of Simulation Optimisation (Amaran et al., 2016), which lends itself well when the problem at hand is stochastic and there is uncertainty or very high complexity of the error surface. Following the proposed approach, we minimise the difference between the target and achieved service measures, given the inventory policy in place, accounting for the inventory position and cost asymmetry of over- and under-stocking. 
Naturally, although the proposed approach aims to address the disconnect between the forecast and inventory management objective, it introduces new complications in the modelling process, which we discuss in this paper.

We evaluate the proposed optimisation on a real case with data from a UK manufacturer of fast moving consumer goods, and demonstrate that the proposed approach results in superior inventory performance, but inferior forecast accuracy compared to conventional cost functions. Given that conventional costs are designed to maximise forecast accuracy, particularly when the cost is of the same order, for example quadratic, this is not unreasonable. This is also in agreement with the observation in the literature, that the most accurate forecast may not always have the best inventory performance (for example see, Kourentzes, 2013, 2014, and references therein). Furthermore, the proposed approach has the advantage that it offers a transparent connection between the parametrisation of the forecasts and their use, which we argue is appealing for practice.

The rest of the paper is organised as follows. Section 2 provides a summary of the background research on the connection between forecast accuracy, estimators and inventory performance. Section 3 outlines the proposed approach, followed by Section 4 that presents the experimental design and the results. Section 5 expands on the results, providing some insights into the implications of using the proposed estimator, followed by concluding remarks in Section 6. 


\section{Research background}

Although evaluation is paramount in the forecasting research, often this is restricted to looking at the forecast accuracy, which is assumed to be a reasonable proxy for the decisions that forecasts support (Ord et al., 2017). Nonetheless, in the literature, there are papers that have questioned this assumption, and arguing for directly using stock control metrics in the context of inventory management (for examples see, Gardner Jr, 1990; Syntetos and Boylan, 2005, 2006; Teunter and Duncan, 2009; Syntetos et al., 2010; Kourentzes, 2013; Syntetos et al., 2015; Sagaert et al., 2018).

Substantial work has been done in the intermittent demand literature, where forecast evaluation is problematic when using conventional error metrics (Kolassa, 2016). Kourentzes (2013) evaluates the use of neural networks for intermittent demand forecasting and finds that although they are inferior in terms of forecast accuracy, ranking almost last amongst the benchmarks considered, the opposite result emerges when looking at inventory performance metrics. Kourentzes (2014) similarly finds that seemingly differently performing methods in terms of accuracy, result in insubstantial differences when evaluated in inventory performance terms. These findings are in agreement with aforementioned references in the intermittent demand literature, raising the question whether forecasts optimal on typical cost functions add considerable value to the subsequent decisions they support, and in particular inventory decisions. Gneiting (2011b) discusses this issue further, pointing that conventional forecast accuracy metrics are effective when we are forecast- 
ing the conditional mean (MSE) or median (MAE), and we should instead consider quantile metrics.

Sanders and Graman (2009) explore the connection between forecasting performance and the resulting decisions in depth. An interesting aspect of this work is that they consider both the accuracy and the bias aspect of forecasting performance. They find that bias has a substantially larger impact on the performance of the subsequent decisions compared to accuracy. For clarity, with the term accuracy we refer to summary statistics that report the magnitude of forecast errors (such as the mean squared and absolute errors), while with the term bias we refer to the under- or over-forecasting of the demand (as captured by the mean error).

It is useful to consider the effect of minimising MSE when generating forecasts. Quadratic loss makes forecasts optimal for the mean of the demand distribution (Gneiting, 2011a). At the same time, the bias-variance decomposition indicates that minimising MSE results in minimising the variance of the forecasts, as well as the in-sample squared bias (defined by the mean error), resulting in in-sample unbiased predictors (Friedman et al., 2001). However, anticipating the out-of-sample performance of MSE optimal forecasts is far from straightforward. For example, Barrow and Kourentzes (2016) provide evidence of how weak the connection is between in-sample and out-of-sample forecast error, where their distributions can differ considerably. They proceed to suggest that forecast combination is a potential remedy. Forecast combination can be perceived as shrinkage operator (Elliott et al., 2013; Kourentzes 
et al., 2018). This connects to the bias-variance trade-off, where the modeller has the challenge of specifying a forecast that balances in-sample over- and under-fitting, given that the true data generating process is unknown, so as to achieve good out-of-sample performance (Friedman et al., 2001). Very accurate in-sample forecasts, and by construction unbiased in-sample if they are optimal on MSE, may be over-fitted. This results in poor out-of-sample performance. Optimising forecast parameters on MSE, or similar cost functions, does not come with any warnings of over-fitting, and it is left up to the modeller to use a variety of model building diagnostics to detect the problem. Therefore, we argue that the challenge of producing well performing forecasts in the out-of-sample, particularly in terms of bias, largely falls on the way we parametrise forecasts. Shrinkage approaches have gained popularity, as they connect model specification and estimation in a single step, and make the bias-variance trade-off evident to the modeller (Tibshirani, 1996; Friedman et al., 2001). This motivates our proposed approach for alternative forecast parametrisation, outlined in the next section.

\section{Optimising directly on inventory performance}

A high level overview of the proposed approach for estimating model parameters is provided in the flowchart in figure 1. This resembles conventional derivative free optimisation, but instead of minimising some historical demand fitting error, at each iteration an inventory simulation is performed, tracking some appropriate performance indicator, which is then used to evalu- 
ate the cost of the optimisation and inform further updating of the forecasting model parameters or stopping the optimisation process.

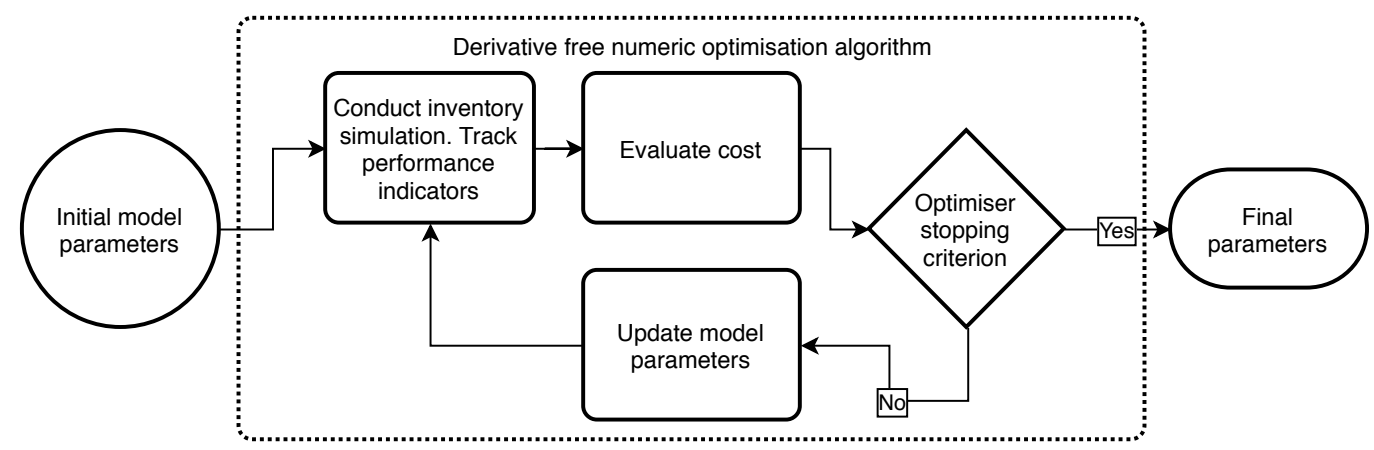

Figure 1: Flowchart of the proposed parameter estimation approach.

It is obvious that the modeller needs to provide the various details for the simulation, such as the relevant inventory policy, lead times, and so on, which are specified so as to match the realistic inventory decision that is faced by the company. In contrast to typical simulation optimisation, in this case there is minimal need for calibration, as inventory policies are well studied processes. Typically the main sources of uncertainty are related to describing the demand process and its uncertainty, which are the forecasting related questions that we are trying to optimise in the first place. Other elements of the simulation are provided by the business context, and depending on the complexity of the business environment and the desired degree of realism, this can often be captured with high certainty. Therefore, contrasting with conventional parameter optimisation using MSE, the only difference in the flowchart in figure 1 is in the top-left cell of the derivative free optimisation. Using MSE we only need to fit the model to the historical data given the 
current parameters prescribed by the optimiser. With the proposed approach we run an inventory simulation using the in-sample data.

It is useful to contrast the sample requirements for conventional time series modelling optimisation and the proposed one in terms of what it implies for the resulting optimal parameters. When estimating the parameters of a forecasting model or method, conventionally, we consider some summary performance statistic, such as the MSE, which becomes the cost function for the optimiser. The MSE is the sample fitting error variance, which estimates the unobserved error variance that we want to minimise, and since it is a random variable we require an adequate sample to obtain a good estimate. At the same time, since the cost function is minimised when the differences between the observed and the predicted values are minimised, this becomes an approximation exercise. As the number of parameters of the forecast function increase, then it can approximate the sample observations with more flexibility. If it correctly describes the underlying data generating process then, since the parameters are random variables themselves, additional sample data increases our confidence in the estimates. If the forecast function has omitted terms, then there are limits to the quality of the approximation, which will become more evident as the sample size increases. Alternatively, if there are additional terms, then the forecast function will over-fit, however this effect will lessen as sample size increases, with the unnecessary parameter estimates eventually converging to zero. In reality, the underlying process is always unknown, and our forecast functions may have 
simultaneously omitted and superfluous terms.

Switching to the proposed simulation based optimisation, we still summarise the performance using a performance metric, which itself is a random variable. From that standpoint, additional sample data will increase the confidence in the estimate, similar to the conventional case. However, the cost function no longer prescribes an approximation exercise to the observed data and, therefore, there is no direct connection between the model terms and the sample size. Indirectly, a reasonable approximation of the data generating process is still required, but one has to note that this is less straightforward than in the conventional case. We argue that since the focus has shifted from model fit to inventory performance, which itself is conditional on variables such as stock on hand and any orders in the system, on top of the observed demand per period. The latter is the only information considered when fitting a model conventionally. This can result in different parameters and resulting forecasts. For example, consider operational production planning, where a consistent forecast across time may be more beneficial than a very accurate, but volatile, forecast (Sagaert et al., 2018; Fildes and Kingsman, 2011). Nonetheless, if the quality of the forecast is very poor, then that impacts negatively on operations. The exact transformation of the forecasts to the decision can be rather complex, or even unknown, depending on the decision. This is precisely when simulation optimisation is beneficial (Amaran et al., 2016).

Specifically for the inventory management case, as the forecasts and their 
variance are used to inform re-ordering decisions and the amount of safety stock, reducing the bias of the forecast becomes more important than accuracy. A forecast that is very accurate in-sample, but gives biased outof-sample forecasts, can negatively impact inventory performance. On the other hand, a less accurate forecast that remains fairly unbiased in the outof-sample can be preferable, even if the forecast function has omitted terms. Similarly, this approach, as it is not minimising the approximation error, will have less tendency to over-fit, even when unnecessary terms are included in the forecast function.

A final important consideration is what should be the appropriate inventory performance metric. Ideally, one should use the overage and underage costs directly, however these are not always easy to obtain, particularly the underage cost. Therefore, we can consider associated metrics, such as the cycle service level and the fill rate. The cost in that case becomes the difference between the target and realised for the sample period. Both the service level and the fill rate blend the overage and the underage cost into a single metric, which effectively reduces a multi-objective optimisation to a conventional single objective one.

In the following section we describe the implementation details of the optimisation, and evaluate the proposed parametrisation against established benchmark approaches, to demonstrate the merit of the idea. 


\section{Empirical evaluation}

\subsection{Case study dataset}

To evaluate the performance of the proposed parametrisation we use a real case study. Our data originate from a UK manufacturing firm that specialises in household cleaning and personal hygiene products. The company serves retailers in the European market and has production facilities distributed across the continent. They operate on a weekly inventory planning cycle and their lead time is typically between 3 and 5 weeks.

Our dataset contains 229 items with 173 weekly sales each. After exploring the sales we did not identify strong evidence of seasonality or trends. We retain the last $m=52$ weeks as a test set, to evaluate the resulting forecasting and inventory performance of the evaluated model parametrisation alternatives.

\subsection{Forecasting setup}

Given the structure of the time series, we use local level exponential smoothing, that is the state space model form of the popular single exponential smoothing method (Hyndman et al., 2002):

$$
\begin{aligned}
\hat{y}_{t+h} & =l_{t}, \quad \text { and } \quad h \geq 1, \\
l_{t} & =l_{t-1}+\alpha \varepsilon_{t},
\end{aligned}
$$


where $\hat{y}_{t+h}$ is the $h$-step ahead forecast from period $t, l_{t}$ the estimate of the local level, $\alpha$ the smoothing parameter and $\varepsilon_{t} \sim N(0, \sigma)$. Observe that the forecast $\hat{y}_{t+h}$ is conditional on the observed sample up to period $t$, the smoothing parameter $\alpha$ and the initial level $l_{0}$, which corresponds to the value of $l_{t-1}$ when $t=1$. The conditional demand over the lead time is simply $\hat{Y}_{t+L}=\sum_{i=1}^{L} \hat{y}_{t+i}$, updated at every period. From the state space framework, we can construct the expression for the conditional variance over the lead time, $L$, given parameter $\alpha$ (Hyndman et al., 2008, p. 92):

$$
V_{L}=\sigma^{2} L\left[1+\alpha(L-1)+\frac{1}{6} \alpha^{2}(L-1)(2 L-1)\right]
$$

Here, $L$ is constant and known, and any review time is included in it. Note that equation (3) does not account for the uncertainty due to the estimation of $\alpha$. Prak et al. (2017) and Prak and Teunter (2019) show that for a small sample size this can lead to high inventory costs, as the total uncertainty is underestimated. However, when there is an adequate estimation sample, the parameter uncertainty becomes sufficiently small to have minuscule impact. Observe that (3) is based on the assumption that forecast errors are normally distributed. Since we are interested in the cumulative demand over the lead time, as the lead time increases, the central limit theorem becomes relevant making the assumption reasonable. Nonetheless, there may be many cases in which the deviations from normality remain strong. In these cases alternative distributions, or empirical approximations, may be preferable. Trapero et al. 
(2019a) and Trapero et al. (2019b) discuss some of these alternatives and the conditions under which they perform well.

We consider a number of alternatives to estimate the smoothing parameter $\alpha$ and the initial level $l_{0}$. Given the state space formulation we can derive the likelihood and maximise it to obtain the optimal parameters given the available sample. This turns out to be equivalent to the well known Mean Squared Error, given the additive nature of the model innovations (Hyndman et al., 2008, p. 69):

$$
\operatorname{MSE}=\frac{1}{n-1} \sum_{t=1}^{n-1}\left(y_{t+1}-\hat{y}_{t+1}\right)^{2},
$$

where $n$ is sample size of the training set and $y_{t}$ the observed demand at period $t$. Kourentzes and Trapero (2018) have argued, echoing similar views by Xia et al. (2011) and Chatfield (2000), that maximising the likelihood is meaningful given the assumption that the underlying model is true for the demand process. This is often adequate for practical considerations. However, when this assumption is strongly violated, minimising (4) will result in model parameters that are adequate only for short term predictions (for a discussion see Chatfield, 2000, Section 6.3). Kourentzes and Trapero (2018) proceed to show that using multi-step cost functions can result in increased forecast accuracy (also see empirical evidence by Clements and Hendry, 1998; Pesaran et al., 2011), and results in a particular form of univariate parameter shrinkage. This also mitigates the parameter estimation uncertainty. One 
can minimise the $h$-steps ahead forecast error, or the average of 1 to $h$-steps ahead error, with the latter enforcing more aggressive shrinkage:

$$
\begin{aligned}
\mathrm{MSE}_{t+h} & =\frac{1}{n-h} \sum_{t=1}^{n-h}\left(y_{t+h}-\hat{y}_{t+h}\right)^{2}, \\
\mathrm{MSE}_{t+1-t+h} & =\frac{1}{h} \sum_{i=1}^{h} M S E_{t+i},
\end{aligned}
$$

Finally, considering that the requirement in inventory management is accurate forecasts over the lead time, one can minimise these directly:

$$
\mathrm{MSE}_{t+L}=\frac{1}{n-L} \sum_{t=1}^{n-L}\left(Y_{t+L}-\hat{Y}_{t+L}\right)^{2}
$$

where actual demand over lead time $Y_{t+L}=\sum_{i=1}^{L} y_{t+i}$.

Finally, the last alternative we consider is to use the inventory simulation approach we propose, where the cost function to be minimised is simply:

$$
\text { Cost }=(p-\hat{p})^{2}
$$

where $p$ is the target cycle service level and $\hat{p}$ is the realised one, for the insample period, based on an inventory simulation for given model parameters.

Given the model expression for the lead time variance (3), we need an estimate for the $\sigma$, which given parameters $\alpha$ and $l_{0}$ is:

$$
\hat{\sigma}=\frac{1}{n-2} \sum_{t=1}^{n}\left(y_{t+1}-\hat{y}_{t+1}\right)^{2} .
$$


We divide by $n-2$ to account properly for the degrees of freedom.

We consider these four estimation approaches, alongside the proposed one, to rigorously benchmark the performance of the inventory simulation optimisation approach. Furthermore, this study provides the opportunity to evaluate the impact of the alternative estimation approaches to the inventory, which has not been done in the literature.

\subsection{Evaluation setup}

We consider lead times of 3 and 5 weeks and perform a rolling origin evaluation. At every period we optimise the model parameters and produce the relevant forecasts. Once this is done, we increase the sample by one observation and repeat. This is repeated until all the test set is used. We consider four forecasting performance metrics, two measuring the magnitude of forecast errors and two the magnitude of bias. For each case, one metric is recording the errors per period, and the other the cumulative errors over lead time.

$$
\begin{aligned}
\mathrm{RMSE}_{h} & =\frac{1}{m-h+1} \sum_{t=n+1}^{n+m-h} \sqrt{\frac{1}{h} \sum_{i=1}^{h}\left(y_{t+i}-\hat{y}_{t+i}\right)^{2},} \\
\mathrm{RMSCE}_{h} & =\frac{1}{m-h+1} \sum_{t=n+1}^{n+m-h} \sqrt{\left(Y_{t+h}-\hat{Y}_{t+h}\right)^{2}}, \\
\mathrm{AME}_{h} & =\mid \frac{1}{m-h+1} \sum_{t=n+1}^{n+m-h}\left(\frac{1}{h} \sum_{i=1}^{h}\left(y_{t+i}-\hat{y}_{t+i}\right)\right) \\
\mathrm{AMCE}_{h} & =\left|\frac{1}{m-h+1} \sum_{t=n+1}^{n+m-h}\left(Y_{t+h}-\hat{Y}_{t+h}\right)\right| .
\end{aligned}
$$


RMSE and AME are calculated on the trace forecast errors from $t+1$ to $t+h$ steps ahead, for each $m-h+1$ forecast origins, where $m$ is the sample size of the test set. Likewise, RMSCE and AMCE are calculated on the difference of the cumulative actuals and forecasts over the lead time, for each forecast origin. Observe that AME and AMCE are measuring the same quantity, as the summations of $y_{t+i}$ and $\hat{y}_{t+i}$ in (12) can be rearranged to give (13). For this reason we only retain AME in the discussion. These errors are scaledependent. We transform them to scale independent by dividing for each time series the resulting metric for estimation method A with a benchmark method B, for which we use the standard 1-step ahead in-sample MSE. These ratios are then summarised across the $q$ time series of the dataset using the geometric mean:

$$
\operatorname{Rel} M_{h}=\left(\prod_{i=1}^{q} \frac{M_{h}^{A}}{M_{h}^{B}}\right)^{\frac{1}{q}}
$$

where $M_{h}=\left(\mathrm{RMSE}_{h}, \mathrm{RMSCE}_{h}, \mathrm{AME}_{h}\right)$. When the value of the relative metric is below one the method A improves over the benchmark by (1 $\left.\operatorname{Rel} M_{h}\right) 100 \%$.

Beyond the forecast performance metrics, we consider the inventory performance of the alternative estimation methods by constructing an inventory simulation. We impose an order-up-to policy and measure the outcome for $p=(90 \%, 95 \%, 99 \%)$ service levels, matching targets in the case study company. The range of $p$ allows us to assess the performance of the proposed optimisation approach in various common settings, given the difficulty in 
estimating implied stock-out and holding costs. The order-up-to level $S$ is calculated as:

$$
S=\hat{Y}_{t+L}+\Phi^{-1}(p) \sqrt{V_{L}}
$$

where $\Phi^{-1}(\cdot)$ is the inverse of the cumulative normal distribution and $V_{L}$ is calculated as in (3).

Note that (15) is the standard approach in an inventory management setting (Silver et al., 2016), where we rely on estimations of the conditional mean and variance of the demand over the lead time. An alternative approach would be to directly estimate the relevant quantile of the cumulative distribution of the demand over the lead time, which can be done either empirically (Trapero et al., 2019a) or by considering modifications in the optimisation of the forecasting models (Gneiting, 2011b). There are conditions which make these alternatives attractive in calculating the order-up-to-level $S$. Given that there is no clear dominance of a single approach, combination approaches have also been investigated (Trapero et al., 2019b). We do not explore these approaches further here, as the conventional formulation in (15) is adequate for our dataset.

We keep track of the realised service level, the stock-on-hand and the stock-out, over the out-of-sample data, scaled by the observed in-sample mean demand, to make these metrics scale independent. We then average these across all time series in the dataset. Any unmet demand is considered lost, which is a reasonable assumption for the products of the case company. 


\subsection{Forecasting performance results}

First, we report the forecasting performance of the competing estimation approaches. Table 1 summarises the various forecasting performance metrics. These are provided at an aggregate level across all time series, for the two different forecast horizons. Note that there are three results for the estimation based on the inventory simulation, one for each target service level. For each horizon and each metric, the best performing estimation method is highlighted in boldface.

Table 1: Forecasting performance metrics across the 229 items

\begin{tabular}{|c|c|c|c|}
\hline \multirow{3}{*}{ Cost function } & \multicolumn{2}{|c|}{ Accuracy } & \multirow{2}{*}{$\frac{\text { Bias }}{\text { RelAME }}$} \\
\hline & RelRMSE & RelRMSCE & \\
\hline & \multicolumn{3}{|c|}{ Horizon 3} \\
\hline MSE & 1.000 & 1.000 & 1.000 \\
\hline $\mathrm{MSE}_{t+h}$ & 0.995 & 0.990 & 1.016 \\
\hline $\mathrm{MSE}_{t+1-t+h}$ & 0.994 & 0.987 & 1.060 \\
\hline $\mathrm{MSE}_{t+L}$ & 0.995 & 0.990 & 1.067 \\
\hline Inventory $(90 \%)$ & 1.033 & 1.086 & 0.678 \\
\hline Inventory $(95 \%)$ & 1.033 & 1.086 & 0.524 \\
\hline \multirow[t]{2}{*}{ Inventory $(99 \%)$} & 1.062 & 1.157 & 0.376 \\
\hline & & Horizon 5 & \\
\hline MSE & 1.000 & 1.000 & 1.000 \\
\hline $\mathrm{MSE}_{t+h}$ & 0.995 & 0.987 & 0.968 \\
\hline $\mathrm{MSE}_{t+1-t+h}$ & 0.991 & 0.974 & 1.043 \\
\hline $\mathrm{MSE}_{t+L}$ & 0.994 & 0.980 & 1.108 \\
\hline Inventory $(90 \%)$ & 1.009 & 1.028 & 0.745 \\
\hline Inventory (95\%) & 1.008 & 1.031 & 0.632 \\
\hline Inventory $(99 \%)$ & 1.023 & 1.089 & 0.473 \\
\hline
\end{tabular}

The results across the two horizons are qualitatively similar. In terms of accuracy, irrespective if we focus at trace (RelRMSE) or cumulative (Rel- 
RMSCE) errors over the horizon, $\mathrm{MSE}_{t+1-t+h}$ performs best, although only marginally compared to the other MSE approaches. When we look at the bias metrics, again for both cases, the inventory based estimation substantially outperforms all MSE based alternatives. The reduction of bias from the conventional MSE ranges between $25 \%$ to $60 \%$. This is achieved with only up to $9 \%$ reduction in accuracy. We anticipate this reduction in bias to have a significant impact on the inventory performance.

Figure 2 provides violin plots of the three forecasting metrics, across time series, for $h=3$. The geometric mean performance for each estimation method is indicated by a horizontal bar. The distributions have been winsorized at $5 \%$ to improve clarity, which also explains the increased concentration of errors at the lower and higher values of the plots. We do not provide the plot for $h=5$, as it is similar qualitatively and does not provide any additional insights. Also note that we provide a single set of results for the inventory based estimator, by calculating the geometric mean across the results for the different target service levels. We do that to avoid cluttering the figure, while losing few insights. Inspecting the figure we can see that the error distributions for both accuracy and bias metrics for the MSE variants are almost identical, and are located around the benchmark MSE results. This indicates that any differences lack significance. On the other hand, the distributions for the inventory based parametrisation are substantially different, with the centre of these distributions significantly differing from the MSE variants. 

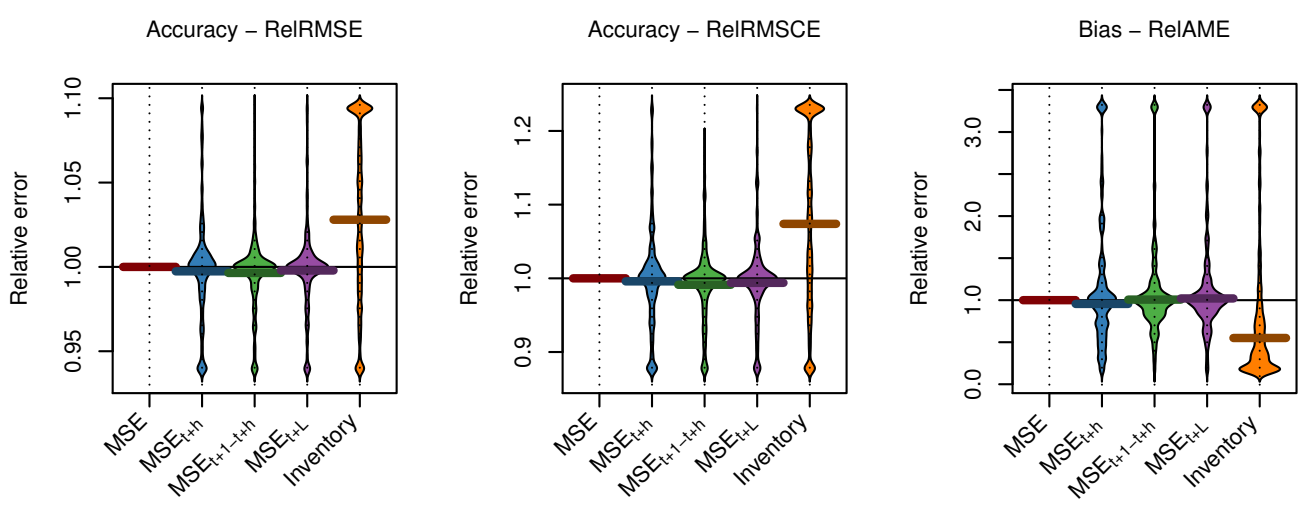

Figure 2: Violin plots of forecasting performance using different optimisation criteria across time series, for $h=3$.

As we previously discussed, the forecasting performance metrics are not well suited to assess the inventory relevant performance of the competing forecasts. In the next subsection we proceed to report on the inventory performance metrics that overcome this limitation.

\subsection{Inventory performance results}

We proceed to explore the performance in terms of inventory by means of trade-off curves (Gardner Jr, 1990; Syntetos et al., 2015), as provided in figures 3 and 4 . The top-left subplot provides the scaled inventory on hand against the service level deviation. We use the deviation to better highlight cases of under- and over-coverage. Ideally, any curves should have zero deviation and as little inventory as possible. Similarly, the top-right plot provides the scaled out of stock against the service level deviation. The bottom-left plot provides the trade-off curve between scaled inventory on 
hand and out of stock volume. In this subplot, the ideal solution would be having zero out of stock, while holding minimal inventory, and therefore any curves closer to the origin $(0,0)$ dominate those more distant.
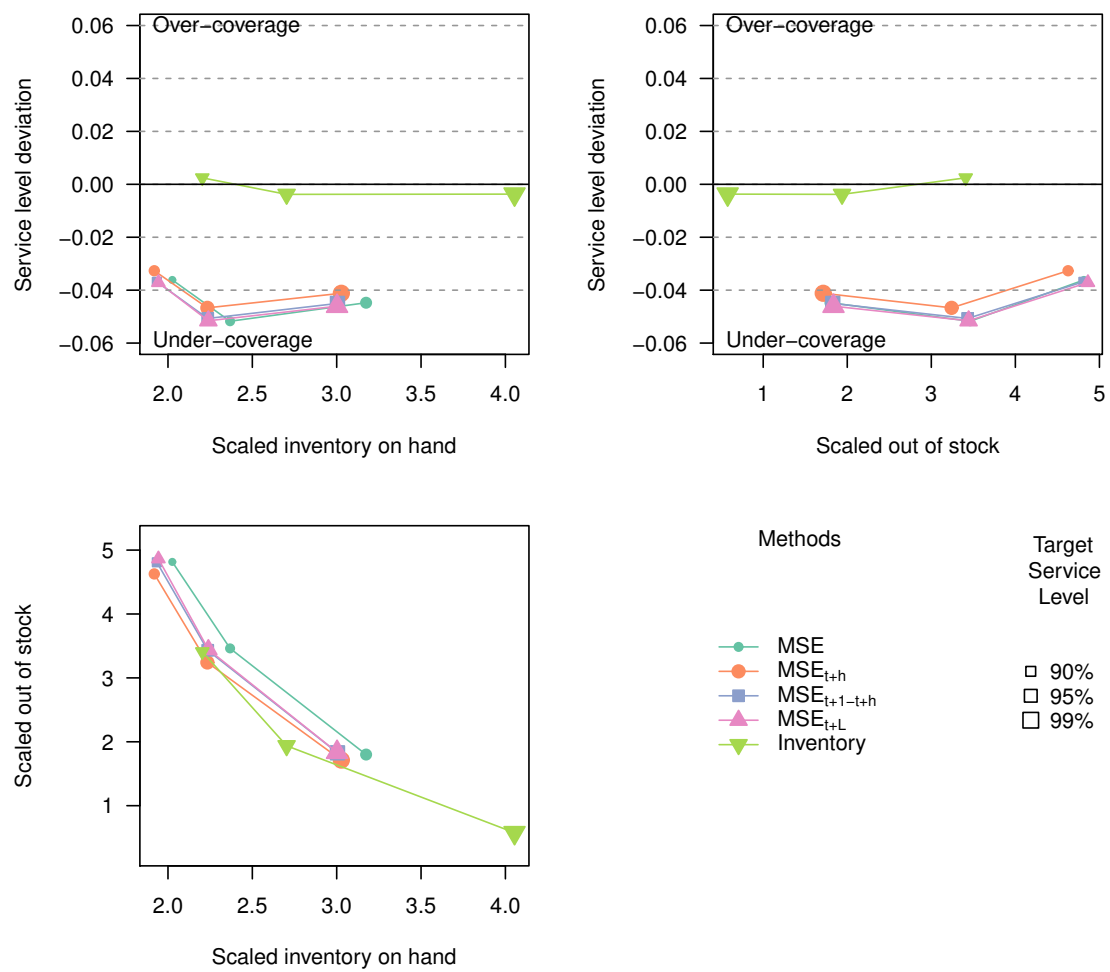

Figure 3: Inventory performance plots for $h=3$.

Both figures 3 and 4 provide similar insights. The proposed inventory simulation based parameter estimation results in the smallest deviation from the target service level, with only limited increase of the stock on hand and reduction of out of stock. This results in dominating trade-off curves over all other estimators. Considering the benchmarks, note that the conventional MSE based optimisation performs the worst, albeit with small differ- 

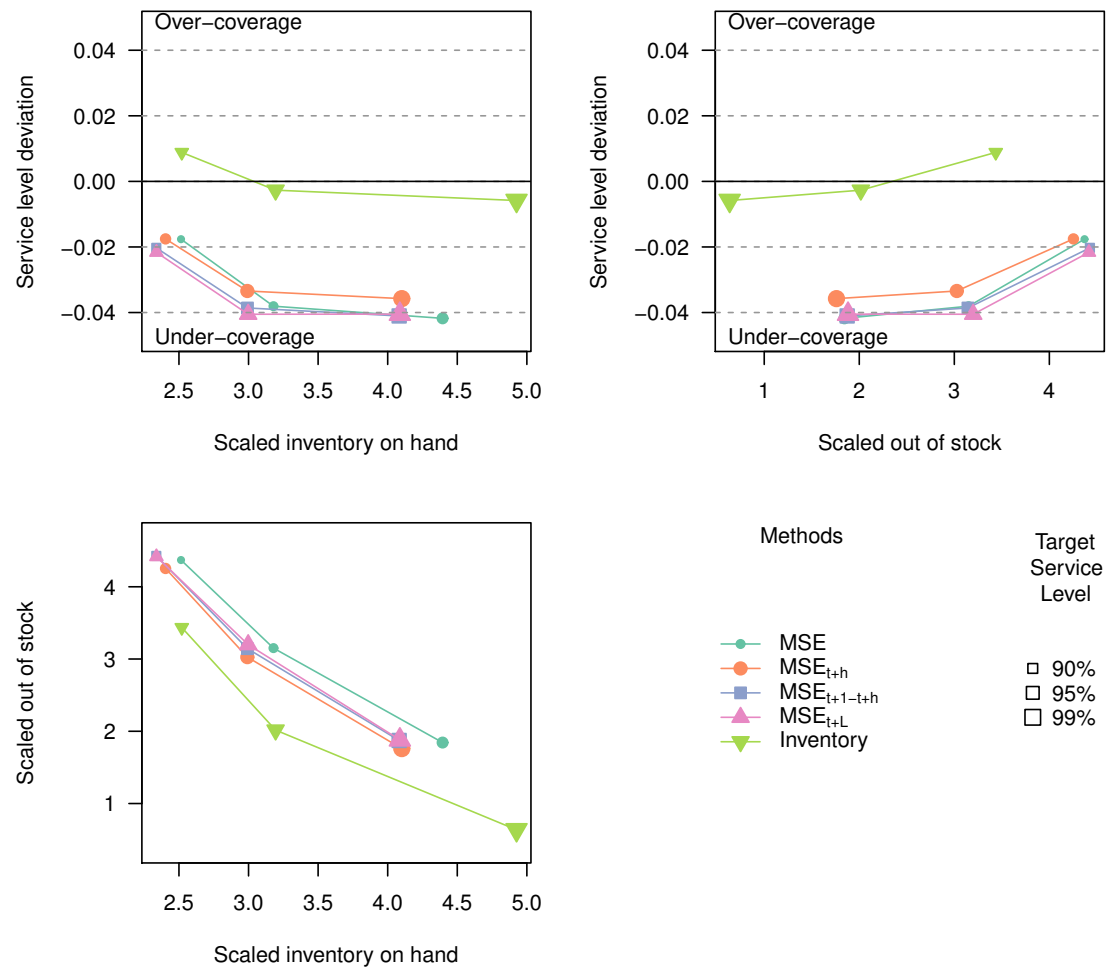

Figure 4: Inventory performance plots for $h=5$.

ences from the remaining MSE variants. The superiority of the proposed parametrisation is stronger for $h=5$.

The inventory performance results follow the discussion of the forecasting performance results, where the substantial gains in terms of out-of-sample bias, with only minimal degradation of accuracy, are translated in superior inventory performance as expected. 


\section{Discussion}

An advantage of MSE-based cost functions is that they typically result in well behaved convex and differentiable error surfaces. In particular for the local level exponential smoothing model they prescribe a globally optimal set of $\alpha$ and $l_{0}$ values, for given historical demand.

It is useful to explore the resulting error surface of the proposed optimisation. We simulate the error surfaces for a time series of our case study, for both MSE and the proposed cost, plotted in figure 5 using contours. The resulting optimal set of parameters are provided as well. Observe how different the two optimal solutions are. Although the MSE error surface is convex and smooth, with a global minimum, this is not the case for the other cost. The inventory based error surface is more complex, non-monotonic and has multiple local minima. This makes the initial starting point of the optimiser important, as poorly chosen sets of starting values can result in low quality solutions. Figure 6 exemplifies this by providing the error surfaces for two different time series, together with multiple starting points for the optimisation and associated end points, which are local minima. In the top subplot we observe that most converge around the same solution. However, in the second case, the large plateaus in the error surface result in many diverse local optima. In fact, many of the poor initialisation points at the right side of the subplot, associated with large $\alpha$ values, are not able to escape the plateau at all. Our approach to avoid these issues was to initialise the optimisation multiple times, as indicated in figure 6 that forces a thorough 
search of the solution space.
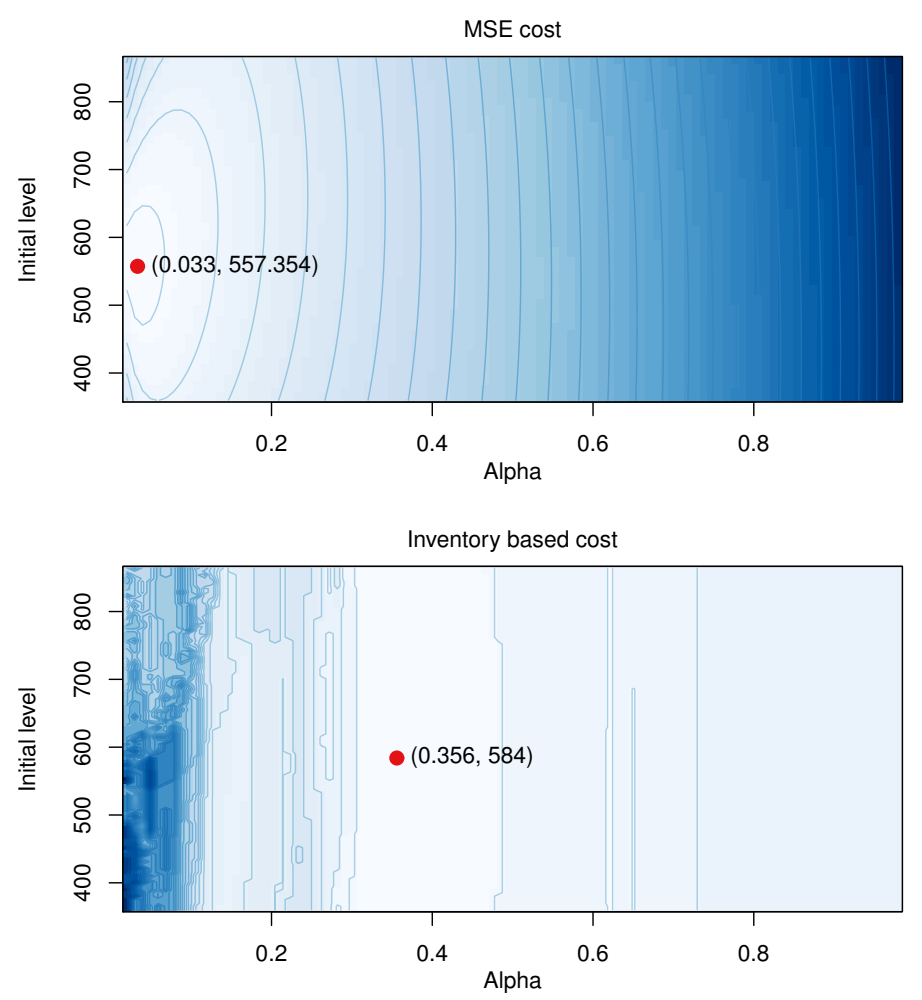

Figure 5: MSE and inventory based error surfaces, together with optimal parameters. Lighter colour denotes lower errors.

Beyond the specifics of the error surface, using an inventory simulation based optimisation has further implications for the forecasting modelling methodology. Although it is applicable to both model and method based forecasts, using models is attractive as they provide analytical variance expressions that are needed for the calculation of safety stocks in an inventory setting. Moreover, using model families is particularly helpful for selecting the appropriate model specification, which is typically done by using infor- 

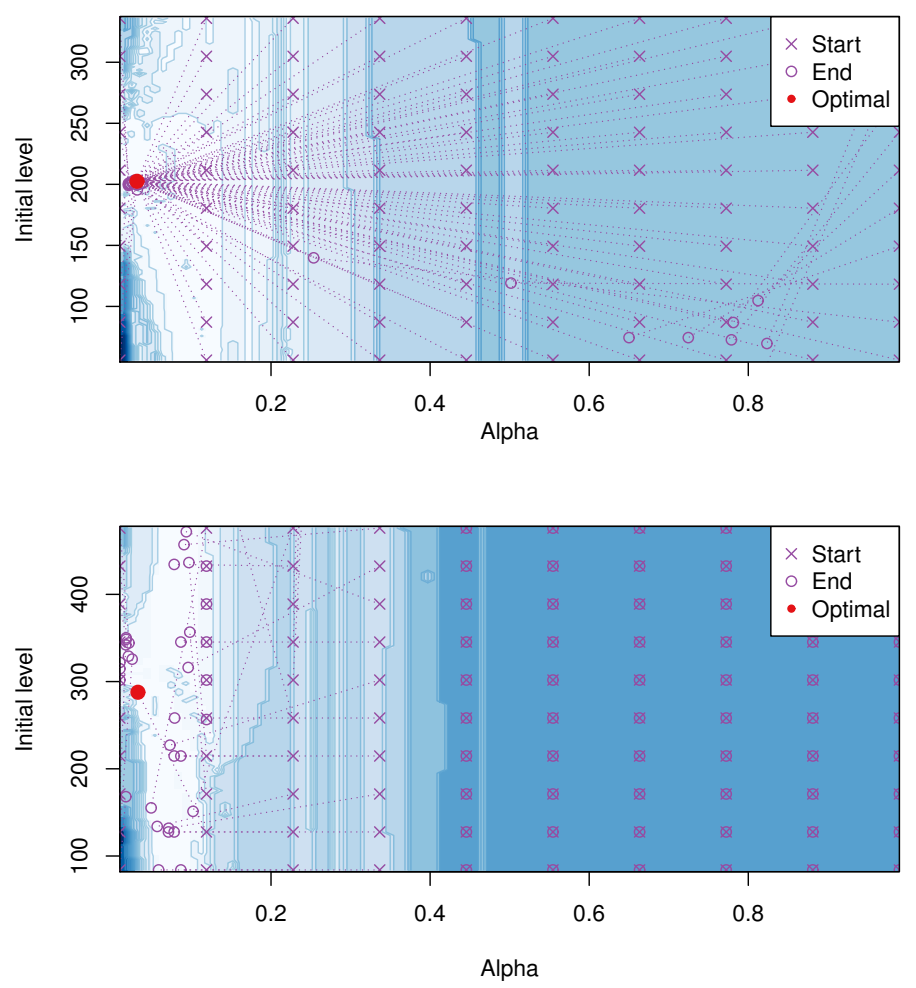

Figure 6: Inventory based error surfaces for two different time series. Multiple optimisation starting points are indicated, together with the corresponding local minima. Lighter colour denotes lower errors.

mation criteria, such as the Akaike Information Criterion (Burnham and Anderson, 2003). However, these require that the likelihood of the model is maximised. This is no longer the case, which makes model selection and specification using information criteria invalid. This limitation is also true for the various other cost functions described in Section 4.2, and more widely in the literature. There are various ways one could overcome this, also applicable when we rely on method based forecasts, such as using cross-validated errors, model combination and selection heuristics (for a discussion and comparison 
of common approaches see Kourentzes et al., 2018), potentially enhanced by domain knowledge (Petropoulos et al., 2018). How to best perform model selection and combination is an open question that requires additional research, with implications for the wider literature.

At this point we should note that the proposed simulation optimisation approach will result in different model parameters, and therefore forecasts, when the settings of the inventory system change. For instance, different target service levels will result in different forecasts. Similarly, if we were to change the various settings of an inventory policy, for example including lost sales or not, this would also impact the resulting forecasts. This is in contrast to the majority of the conventional forecast selection methodologies, which are focused on the forecasted conditional mean. This is irrespective of whether we are selecting a forecast using cross-validation, information criteria (Kourentzes et al., 2019) or arguably even judgement (Petropoulos et al., 2018). We argue that this is a benefit of the purposed optimisation approach, as it considers the various feedback loops in the inventory policy, tied to the stock levels, the implicit over- and underage costs and so on that are omitted by the conventional approaches.

\section{Conclusions}

In this paper we proposed to parametrise forecasting models using a cost function inferred directly from the inventory decisions, instead of minimising the fitting error on past historical demand, as is the norm. We found that 
this resulted in lower forecast accuracy than conventional quadratic loss functions, which is not surprising. The loss in terms of accuracy was as high as $9 \%$. However, there were substantial gains in terms of out-of-sample forecast bias, of up to $62 \%$ improvement. When translated to inventory performance, the proposed cost function achieved minimal differences between the target and realised service levels, in contrast to the benchmarks. Furthermore, the resulting out of stock-inventory on hand trade-off curves dominated other alternatives.

The inventory gains come at a cost of a more difficult optimisation problem. We demonstrate that the resulting error surfaces have multiple local minima and large plateaus, and suggested using multiple starting points for the optimiser to achieve an effective search of the solution space. It is of interest to explore alternative optimisation strategies that may result in a more efficient parameter space search.

An attractive property of the simulation optimisation approach that we propose for setting the parameters of the forecasting models is that we can incorporate a wide variety of inventory management setups. For instance, in our case company we considered any unmet demand to be lost. This can easily be modified as needed for different cases. Similarly, while we used an order-up-to policy, this is not a necessity and different policies can be incorporated. Focusing on demonstrating the efficacy of the proposed parameter optimisation approach, we retained many of the classical elements of the order-up-to policy, such as assuming normally distributed forecast 
errors and relying on the analytical formulas for the variance of the demand over the lead time. Again, these can be relaxed and modified to incorporate other distributional assumptions or empirical estimates.

In this case study, given the available data, there was no need to complicate the modelling process by considering alternative exponential smoothing model forms. Nonetheless, the proposed optimisation makes the use of information criteria invalid and one has to revert to more generally applicable strategies, such as using cross-validated routines. Future research should explore whether the proposed approach can facilitate novel model selection strategies, driven directly by the relevant decision being simulated. At this point it is useful to note that as the model parameters are not optimised based on how well the conditional mean of the forecast fits to the observed demand, but rather on the resulting inventory performance, we do not need to focus on the standard outputs of the forecasting model, but instead directly use the elements of the predictive distribution that are more convenient for the implemented inventory policy.

Finally, here we consider the inventory decision to construct the appropriate simulation based cost function. An attractive property of this modelling question is that inventory decisions are high frequency, and therefore occur multiple times within the in-sample period, thus providing adequate sensitivity for the optimisation. However, with the proposed approach we can exchange the inventory decision with other problems as needed. Therefore, it provides a general framework for how to parametrise forecasts directly on 
the decisions, as long as an associated simulation can be constructed. Exploring further the generality of the approach for parametrising forecasts in other decision making contexts will be valuable.

\section{Acknowledgement}

This work was partially supported by the European Regional Development Fund and Spanish Government (MINECO/FEDER, UE) under the project with reference DPI2015-64133-R.

\section{References}

Amaran, S., Sahinidis, N. V., Sharda, B., Bury, S. J., 2016. Simulation optimization: a review of algorithms and applications. Annals of Operations Research 240 (1), 351-380.

Barrow, D. K., Kourentzes, N., 2016. Distributions of forecasting errors of forecast combinations: implications for inventory management. International Journal of Production Economics 177, 24-33.

Burnham, K. P., Anderson, D. R., 2003. Model selection and multimodel inference: a practical information-theoretic approach. Springer Science \& Business Media.

Chatfield, C., 2000. Time-series forecasting. Chapman and Hall/CRC.

Clements, M., Hendry, D., 1998. Forecasting economic time series. Cambridge University Press. 
Elliott, G., Gargano, A., Timmermann, A., 2013. Complete subset regressions. Journal of Econometrics 177 (2), 357-373.

Fildes, R., Kingsman, B., 2011. Incorporating demand uncertainty and forecast error in supply chain planning models. Journal of the Operational Research Society 62 (3), 483-500.

Friedman, J., Hastie, T., Tibshirani, R., 2001. The elements of statistical learning, 10th Edition. Vol. 1. Springer series in statistics New York, NY, USA:

Gardner Jr, E. S., 1990. Evaluating forecast performance in an inventory control system. Management Science 36 (4), 490-499.

Gardner Jr, E. S., 2006. Exponential smoothing: The state of the art-part II. International journal of forecasting 22 (4), 637-666.

Gneiting, T., 2011a. Making and evaluating point forecasts. Journal of the American Statistical Association 106 (494), 746-762.

Gneiting, T., 2011b. Quantiles as optimal point forecasts. International Journal of forecasting $27(2), 197-207$.

Hyndman, R., Koehler, A. B., Ord, J. K., Snyder, R. D., 2008. Forecasting with exponential smoothing: the state space approach. Springer Science \& Business Media. 
Hyndman, R. J., Koehler, A. B., Snyder, R. D., Grose, S., 2002. A state space framework for automatic forecasting using exponential smoothing methods. International Journal of forecasting 18 (3), 439-454.

Kolassa, S., 2016. Evaluating predictive count data distributions in retail sales forecasting. International Journal of Forecasting 32 (3), 788-803.

Kourentzes, N., 2013. Intermittent demand forecasts with neural networks. International Journal of Production Economics 143 (1), 198-206.

Kourentzes, N., 2014. On intermittent demand model optimisation and selection. International Journal of Production Economics 156, 180-190.

Kourentzes, N., Barrow, D., Petropoulos, F., 2018. Another look at forecast selection and combination: evidence from forecast pooling. International Journal of Production Economics.

Kourentzes, N., Barrow, D., Petropoulos, F., 2019. Another look at forecast selection and combination: Evidence from forecast pooling. International Journal of Production Economics 209, 226-235.

Kourentzes, N., Trapero, J. R., 2018. On the use of multi-step cost functions for generating forecasts. Department of Management Science Working Paper Series.

Ord, J. K., Fildes, R., Kourentzes, N., 2017. Principles of Business Forecasting, 2nd Edition. Wessex Press Publishing Co. 
Pesaran, M. H., Pick, A., Timmermann, A., 2011. Variable selection, estimation and inference for multi-period forecasting problems. Journal of Econometrics 164 (1), 173-187.

Petropoulos, F., Kourentzes, N., Nikolopoulos, K., Siemsen, E., 2018. Judgmental selection of forecasting models. Journal of Operations Management.

Prak, D., Teunter, R., 2019. A general method for addressing forecasting uncertainty in inventory models. International Journal of Forecasting 35 (1), 224-238.

Prak, D., Teunter, R., Syntetos, A., 2017. On the calculation of safety stocks when demand is forecasted. European Journal of Operational Research $256(2), 454-461$.

Sagaert, Y. R., Kourentzes, N., De Vuyst, S., Aghezzaf, E.-H., Desmet, B., 2018. Incorporating macroeconomic leading indicators in tactical capacity planning. International Journal of Production Economics.

Sanders, N. R., Graman, G. A., 2009. Quantifying costs of forecast errors: A case study of the warehouse environment. Omega 37 (1), 116-125.

Silver, E. A., Pyke, D. F., Thomas, D. J., 2016. Inventory and production management in supply chains. CRC Press.

Syntetos, A. A., Babai, M. Z., Gardner Jr, E. S., 2015. Forecasting intermittent inventory demands: simple parametric methods vs. bootstrapping. Journal of Business Research 68 (8), 1746-1752. 
Syntetos, A. A., Boylan, J. E., 2005. The accuracy of intermittent demand estimates. International Journal of forecasting 21 (2), 303-314.

Syntetos, A. A., Boylan, J. E., 2006. On the stock control performance of intermittent demand estimators. International Journal of Production Economics 103 (1), 36-47.

Syntetos, A. A., Nikolopoulos, K., Boylan, J. E., 2010. Judging the judges through accuracy-implication metrics: The case of inventory forecasting. International Journal of Forecasting 26 (1), 134-143.

Teunter, R. H., Duncan, L., 2009. Forecasting intermittent demand: a comparative study. Journal of the Operational Research Society 60 (3), 321329.

Tibshirani, R., 1996. Regression shrinkage and selection via the lasso. Journal of the Royal Statistical Society. Series B (Methodological), 267-288.

Trapero, J. R., Cardos, M., Kourentzes, N., 2019a. Empirical safety stock estimation based on kernel and garch models. Omega 84, 199-211.

Trapero, J. R., Cardós, M., Kourentzes, N., 2019b. Quantile forecast optimal combination to enhance safety stock estimation. International Journal of Forecasting 35 (1), 239-250.

Xia, Y., Tong, H., et al., 2011. Feature matching in time series modeling. Statistical Science 26 (1), 21-46. 